\title{
Controle de qualidade de vinagres comercializados em Manaus
}

\author{
Quality control of vinegars marketed in Manaus \\ Control de calidad de los vinagres comercializados en Manaus
}

Recebido: 06/11/2021 | Revisado: 12/11/2021 | Aceito: 13/11/2021 | Publicado: 15/11/2021

\author{
Nistefanny Santana de Souza \\ ORCID: https://orcid.org/0000-0002-9179-0604 \\ Universidade Nilton Lins, Brasil \\ E-mail: nistefannysantana033@gmail.com \\ Layla Talissa Tundis Pereira \\ ORCID: https://orcid.org/0000-0003-3629-0161 \\ Faculdade Dom Alberto, Brasil \\ E-mail: talissalayla@gmail.com \\ Gabriel Oliveira de Souza \\ ORCID: https://orcid.org/0000-0001-9168-074X \\ Universidade Nilton Lins, Brasil \\ E-mail: gabriel.souza@uniniltonlins.edu.br
}

\begin{abstract}
Resumo
O vinagre caracteriza-se como uma solução diluída a base de ácido acético, estabelecida por dois processos: a fermentação alcoólica, quando o açúcar é modificado para etanol; e a oxidação fermentativa, convertendo o álcool em ácido acético. Com isso, ao longo da história, ele vem sendo usado como condimento e conservante de alimentos, além de ser constituído por propriedades nutritivas e biorregulatórias apontadas como complementos fundamentais na alimentação. O vinagre pode manifestar efeitos positivos fisiológicos relacionado com a regulação pressão arterial, controle da glicose sanguínea, auxílio na digestão e estimulação do apetite. O objetivo do estudo foi avaliar a qualidade de amostras de vinagres comercializados em Manaus. Foram selecionadas aleatoriamente 5 amostras de vinagres de diferentes marcas identificadas como amostras A, B, C, D, E, encontradas em estabelecimentos comerciais da cidade de Manaus-AM, no período de setembro a outubro de 2021. O teor de ácido acético nos vinagres foi quantificado seguindo metodologia adaptada de Baccan, 2001. O teor do ácido acético quantificado nos vinagres variou de $3,08 \%$ a 4,57\%. As amostras C, D e E apresentaram valores de acidez abaixo do padrão estabelecido, os demais apresentaram valores de acidez de acordo com o que preconiza a legislaçãoa legislação vigente.
\end{abstract}

Palavras-chave: Ácido acético; Composição fenólica; Controle de qualidade; Fermentação; Vinagre.

\begin{abstract}
Vinegar is characterized as a dilute solution based on acetic acid, established by two processes: alcoholic fermentation, when sugar is changed to ethanol; and fermentative oxidation, converting alcohol into acetic acid. Thus, throughout history, it has been used as a condiment and food preservative, in addition to being constituted by nutritional and bioregulatory properties identified as fundamental complements in food. Vinegar can manifest positive physiological effects related to blood pressure regulation, blood glucose control, aid in digestion and appetite stimulation. The aim of the study was to evaluate the quality of samples of vinegars sold in Manaus. Five samples of vinegars of different brands identified as samples A, B, C, D, E, found in commercial establishments in the city of Manaus-AM, from September to October 2021, were randomly selected. The acetic acid content in vinegars was quantified following methodology adapted from Baccan, 2001. The acetic acid content quantified in vinegars ranged from $3.08 \%$ to $4.57 \%$. Samples C, D and E presented acidity values below the established standard, the others presented acidity values in accordance with what is recommended by the legislation in force. Keywords: Acetic acid; Phenolic composition; Quality control; Fermentation; Vinegar.
\end{abstract}

\section{Resumen}

El vinagre se caracteriza por ser una solución diluida a base de ácido acético, que se establece mediante dos procesos: fermentación alcohólica, cuando el azúcar se transforma en etanol; y oxidación fermentativa, que convierte el alcohol en ácido acético. Así, a lo largo de la historia, se ha utilizado como condimento y conservante de alimentos, además de estar constituido por propiedades nutricionales y biorreguladoras identificadas como complementos fundamentales en los alimentos. El vinagre puede manifestar efectos fisiológicos positivos relacionados con la regulación de la presión arterial, el control de la glucosa en sangre, la ayuda en la digestión y la estimulación del apetito. El objetivo del estudio fue evaluar la calidad de las muestras de vinagres vendidos en Manaus. Se seleccionaron aleatoriamente cinco muestras de vinagres de diferentes marcas identificadas como muestras A, B, C, D, E, encontradas en establecimientos comerciales de la ciudad de Manaus-AM, de septiembre 
a octubre de 2021. Se cuantificó el contenido de ácido acético en los vinagres siguiendo la metodología adaptada de Baccan, 2001. El contenido de ácido acético cuantificado en vinagres osciló entre 3,08\% y 4,57\%. Las muestras C, D y E presentaron valores de acidez por debajo del estándar establecido, las demás presentaron valores de acidez de acuerdo con lo recomendado por la legislación vigente.

Palabras clave: Ácido acético; Composición fenólica; Control de calidad; Fermentación; Vinagre.

\section{Introdução}

O vinagre caracteriza-se como uma solução diluída de ácido acético, estabelecida por dois processos: a fermentação alcoólica, quando o açúcar é modificado para etanol; e a oxidação fermentativa, convertendo o álcool em ácido acético. Com isso, ao longo da história, ele vem sendo usado como condimento e conservante de alimentos, além de ser constituído por propriedades nutritivas e que são apontadas como complementos fundamentais na alimentação (Costa, 2017). Além disso, o vinagre pode manifestar efeitos positivos fisiológicos relacionado ao controle da pressão arterial, regulação da glicose sanguínea, auxílio na digestão e estimulação do apetite (Marques et al., 2010).

Hoje se sabe que fermentação acética que produz o vinagre é devido a presença de bactérias do gênero Acetobacter ou Gluconobacter, perttencentes à família Pseudomonoceae (Mota, 2011). A principal finalidade deste condimento é atribuir gosto e aroma aos alimentos (Oliveira, 2014).

O ácido acético contido no vinagre foi durante séculos, o ácido mais forte existente, e este era utilizado, mesmo sem conhecer seu príncipio ativo, por soldados romanos, que recebiam um pequeno volume de vinagre para que durante suas viagens usassem na água antes de ingeri-la, evitando assim doenças provocadas por águas contaminadas (Maestre, 2017).

Com a descoberta dos benefícios do vinagre, o mesmo começou a ter uma comercialização mundial, e com ela, o avanço de novas tecnologias que beneficiasse a produção de ácido acético. Essa tendência de consumo gera o desenvolvimento de novos produtos os quais expandem a variedade de vinagres disponíveis no mercado (Dutra, 2021).

O vinagre é um produto de acessibilidade fácil no Brasil, porém pouco valorizado no comércio, em razão do desconhecimento do consumidor diante de suas propriedades funcionais. Isso se reflete em virtude do número mínimo de pesquisas realizadas no país sobre essa temática (Schmoeller, Balbi, 2010).

Cerca de 170 milhões de litros de vinagres são consumidos anualmente no Brasil, sendo que 80\%são referentes ao vinagre de álcool. Depois deste, o produto elaborado à base de vinho é o mais consumido pela população brasileira. Embora o termo vinagre, isoladamente, corresponda ao produto obtido da acetificação do vinho, a matéria-prima utilizada para sua elaboração é variável em função da disponibilidade de cada país em que é fabricado, podendo partir do vinho, arroz, sidra, malte, maçã, ácool (Kropzak, Eugenia, 2010).

O alto consumo de vinagre em nosso país deve-se ao fato do Brasil ser um país tropical, onde o consumo de salada é incentivado pelo calor, e a consequente necessidade de uma alimentação mais leve, mais saudável, estimulando o cuidado com a saúde. O vinagre pode ser usado no tempero de saladas, mas também na limpeza dos legumes e verduras, mostrandoa sua açãobactericida (Boffo, 2004).

Quando o vinagre é comercializado por intermédio de frutas, a legislação brasileira recomenda que o produto precisa ser intitulado como vinagre de fruta ou fermentado acético de fruta, assim como também não deve ser utilizado os dois termos, pois são equivalentes . Quanto às características físico-químicas do fermentado acético deverão estar em concordância com a composição do produto (Lima, 2014).

A acidez do vinagre desempenha intervenções na aceitação sensorial do produto, sendo o percentual de ácido acético dos vinagres correspondente à acidez percebida de maneira sensorial (Cordeiro; Fregona; Leão, 2019).

Contudo, é proibido também a adição de corantes em vinagres, como caramelo, o uso de melaço, subproduto do açúcar, mesmo como nutriente, na elaboração do vinagre. O uso de aditivos, conservante ou nutrientes só poderá ocorrer mediante prévia autorização do órgão competente (Fiorio; Dalposso, 2011). 
O vinagre é um produto de fácil acesso no Brasil, podendo ser adquiridos desde tabernas a grandes supermecados, entretando ainda é pouco valorizado comercialmente devido, em grande parte pela população ainda desconhecer suas propriedades funcionais. Isso reflete no pequeno número de pesquisas realizadas no país sobre este tema. Por tanto, nossa pesquisa tem o objetivo de avaliar a qualidade dos vinagres comercializados em Manaus e dessa formas comparar coma lesgilação quanto ses parametros de qualidade.

\section{Metodologia}

O estudo desenvolvido trata-se de uma pesquisa laboratorial de natureza quantitativa, com metodologia baseada em Baccan, 2001. A analises das amostras escolhidas de forma aleatória de diferentes marcas de vinagres identificadas como amostras A, B, C, D, E, encontradas em supermercados na cidade de Manaus-AM, no período de setembro a outubro de 2021. As análises foram realizadas em triplicata e os resultados foram expressos em $\mathrm{g} \%$ de vinagres. A Determinação da acidez total está apresentada na Figura 1.

Iniciou-se o procedimento adaptado de Baccan, 2001, transferindo $20,0 \mathrm{~mL}$ da solução de vinagre puro $4 \%$ para um béquer médio. Em seguida transferiu-se essa solução para um balão volumétrico de $100 \mathrm{ml}$ e completou o volume para 100,0 $\mathrm{mL}$ com água destilada e agitou-se vigorosamente por três vezes essa amostra para uma melhor homogeneização. Com a utilização de uma pipeta graduada de $10,0 \mathrm{~mL}$ e $5,0 \mathrm{~mL}$ transferiu-se um volume de $25,0 \mathrm{~mL}$ da solução preparada para um Erlenmeyer e com o auxílio de uma proveta adicionou-se $50,0 \mathrm{~mL}$ de água destilada e posteriormente, 3 gotas de fenolftaleína. A solução titulante foi de $\mathrm{NaOH}$ 0,1 molar. Procedeu-se com a titulação e observou-se o volume consumido de $\mathrm{NaOH}$ e anotouse para posterior cálculo de cada amostra.

Os volumes gastos de hidróxido de sódio e o erro das amostras estão descritos na Tabela 1. Ressalta-se que esse processo foi realizado em triplicata para cada tipo de vinagre e os cálculos foram repetidos para todas as amostras. Com esses valores anotados pôde-se calcular o teor de acidez, ou seja, a porcentagem de ácido acético presente no vinagre (Gusmão et al, 2020).

Figura 1: Fluxograma da metodologia de análise para determinação do teor de ácido acético nas amostras de vinagres.
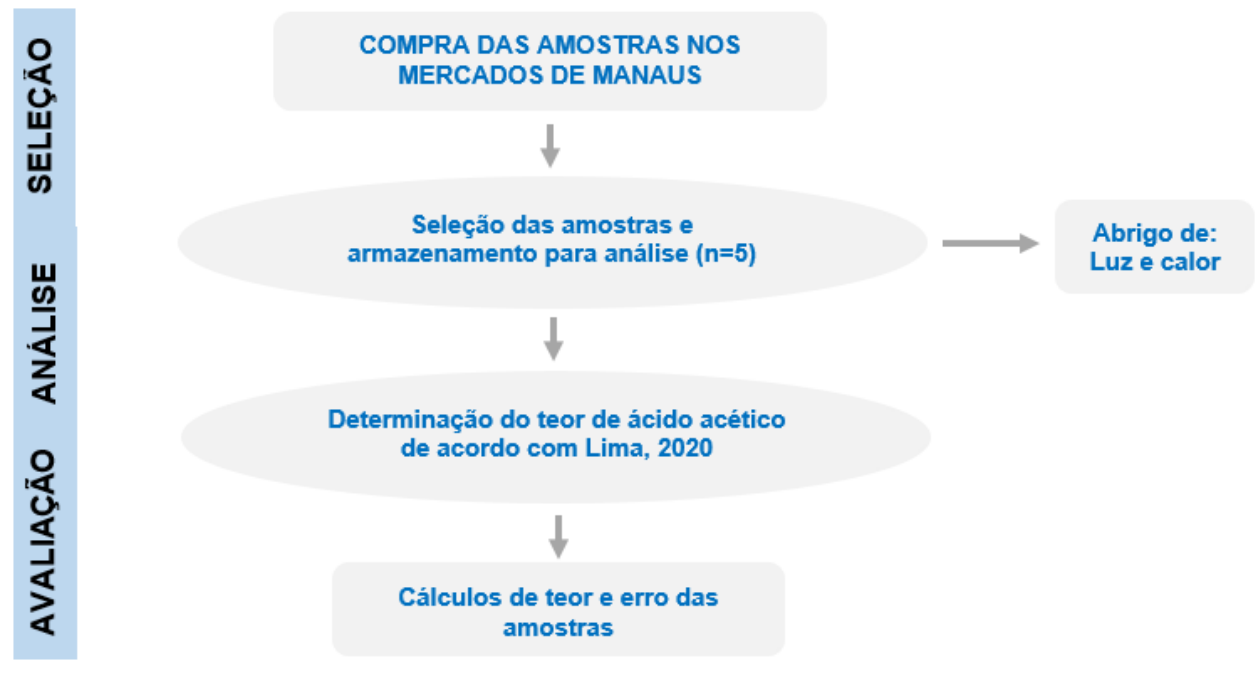

Fonte: Autores.

O teor de ácido acético foi determinado utilizando-se a equação abaixo (Baccan, 2001):

$$
\text { Teor de Ácido Acético }=\frac{[] \mathrm{NaOH} * \mathrm{Vde} \mathrm{NaOH} * \mathrm{MM} \text { do } \mathrm{CH} 3 \mathrm{COOH}}{V \text { de } \mathrm{NaOH}(\mathrm{am}) * \mathrm{VNaOH}(\text { puro })} * 100
$$


Onde:

[ ] NaOH = Concentração da solução de hidróxido de Sódio;

$\mathrm{V} \mathrm{NaOH}=$ Volume de hidróxido de Sódio usado na titulação;

$\mathrm{MM}$ de $\mathrm{CH}_{3} \mathrm{COOH}=$ Massa molar de hidróxido de Sódio;

Por fim, os resultados foram analisados estatisticamente a fim de determinar o erro das análises realizadas em triplicata e comparadas com os valores preconizados pela legislação vigente (Brasil, 1999).

\section{Resultados e Discussão}

A determinação do teor de ácido acético em vinagres é feita por volumtria de neutralização com solução de hidroxido de sódio diluida , como demonstra a equação a seguir:

$$
\mathrm{CH}_{3} \mathrm{COOH}_{(\mathrm{aq})}+\mathrm{NaOH}_{(\mathrm{aq})} \rightarrow \mathrm{NaCH}_{3} \mathrm{COO}_{(\mathrm{aq})}+\mathrm{H}_{2} \mathrm{O}
$$

Aplicando a fórmula do teor de acidez, considerando o volume gasto de $\mathrm{NaOH} 0,1 \mathrm{~mol} / \mathrm{L}$, os resultados obtidos foram $4,57 \%, 4,14 \%, 3,08 \%, 3,40 \%$ e 3,15\%. Onde todos valores são oriundos de triplicadas e analises. Levando em consideração o resultado obtido pode-se comprovar que o teor de ácido acético para o consumo humano está próximo do padrão de referencias da Embrapa, 4 e 6\%(Mindelo et al, 2019). A legislação nacional vigente que trata do teor de ácido acético, determina um valor mínimo de 4,00\% de ácido acético em vinagres (Brasil, 1999). Na Tabela 1, pode-se avaliar o teor do ácido acético encontrado nas amostras de vinagres que variou de $3,08 \%$ a $4,57 \%$.

Tabela 1: Resultado das análises de teor de acidez das amostras. Manaus (2021).

\begin{tabular}{ccccc}
\hline AMOSTRA DE VINAGRES & TIPO DE VINAGRE & $\begin{array}{c}\text { VOLUME GASTO E } \\
\text { ERRO PADRÃO }\end{array}$ & $\begin{array}{c}\text { ACIDEZ- } \\
\text { RÓTULO \% }\end{array}$ & $\begin{array}{c}\text { ACIDEZ DAS } \\
\text { AMOSTRAS } \\
\text { AVALIADAS\% }\end{array}$ \\
\hline Amostra A & Maçã & $38,1 \pm 0,070$ & 4,0 & 4,57 \\
Amostra B & Tinto & $34,5 \pm 0,035$ & 4,0 & 4.14 \\
Amostra C & Arroz & $25,7 \pm 0,100$ & 4,0 & 3,08 \\
Amostra D & Álcool & $28,4 \pm 0,106$ & 4,0 & 3,40 \\
Amostra E & Branco & $26,3 \pm 0,072$ & 4,0 & 3,15 \\
\hline
\end{tabular}

Fonte: Autores (2021).

De acordo com as analisadas, as amostras de vinagres $\mathrm{C}, \mathrm{D}$, e E apresentaram valores de acidez abaixo do padrão estabelecido. Segundo a comparação entre os dados fornecidos pelos fabricantes apresentado no rótulo do produto e os dados obtidos experimentalmente demonstrados na tabela 1, somente as amostras de vinagre A e B obtiveram o teor de ácido acético conforme a Legislação.Esses vinagres com teor de ácido acético acima de $4 \%$ podem ser submetidos a um processo de diluição, para obter-se um maior rendimento.

De acordo com Machado et al. (2019), o teor de álcool fermentado representa o resíduo do processo de acetificação. Todo vinagre deve ter um pouco de álcool, caso contrário as bactérias acéticas, na ausência de um substrato alcoólico, podem degradar o ácido acético produzido com prejuízo para o próprio vinagre.

Verifica-se ainda que dentre as amostras analisadas três delas possuem um teor de ácido acético inferior que o permitido pela legislação, isso se deve, possivelmente a fatores como: erros de analise na execução das técnicas de determinação de teor de ácido acético em amostras, ou a possíveis alterações por parte dos fabricantes. O que nesse caso estaria em desacordo com a legislação vigente (Mindelo et al, 2019). 
A insuficiência de açúcar na fermentação, uma vez que muitos agricultores não têm o habito de corrigir o teor de açúcar como matéria prima para elaboração do vinagre. Em ambos os casos, a matéria prima de partida apresentará um baixo teor alcoólico, e consequentemente um baixo teor de ácido acético (Venquiaruto et al, 2016).

A baixa produção de ácido acético pode ocorrer também devido à conversão inadequada de etanol em acetaldeído ou indisponibilidade de microrganismos envolvidos no mecanismo de fermentação durante a produção do vinagre (Viroli et al., 2021).

\section{Conclusão}

Na presente pesquisa foram analisadas cinco amostras de diferentes marcas de vinagres comercializados, com o intuito de verificar o teor de ácido acético presente nas mesmas. Das cinco amostras, verificou-se que duas amostras com valores de 4,14\% e 4,57\% apresentaram um teor de ácido acético compatível com os padrões estabelecidos pela legislação vigente no Brasil, a qual estabelece um teor de ácido acético mínimo de $4 \%$.

Desse modo a verificação das características físico-químicas dos vinagres comercializados na cidade de Manaus, apresentou-se em sua maioria um teor insatisfatório com valores de 3,08\%, 3,40\% e 3,15\% no qual é fora dos padrões da legislação, como resultado obtido pode-se dizer que todos os demais estão no padrão minimo indicado, entretanto, nenhuma das amostras estavam de acordo com a sua rotulagem, sendo que em todas as amostras avaliadas continha em seu rótulo o teor de ácido acético de $4 \%$.

Além disso o procedimento empregado é um método simples que pode ser aplicado para avaliação da veracidade dos teores de acidez presente no vinagre, produto consumido em grande escala pelas famílias brasileiras. É um instrumento de interesse para os órgãos fiscalizadores de vigilância. O que destaca-se a importância dessa pesquisa, pois através desta foi possível, avaliar a qualidade referente ao teor de ácido acético nos vinagres, além de contribuir para elaboração de futuras pesquisas, destacando-se que existem poucos estudos na literatura sobre essa temática.

\section{Referências}

Baccan, N., Andrade, J. C., Godinho, O. E. S. \& Barone, J. S. (2001). Química analítica quantitativa elementar. (3a ed.), Edgard Blücher.

Boffo E.F. (2004). Estudo da origem biossintética do ácido acético e determinação da acidez em amostras de vinagres comerciais. 68 p. Dissertação (mestrado)Universidade Federal de São Carlos, Centro de ciências exatas e de tecnologia, Programa de Pós-Graduação em Química. São Paulo. CDD: 547.35 (20a).

Brasil. Instrução Normativa (1999) n. 36. Aprova o regulamento técnico para fixação dos padrões de identidade e qualidade para fermentados acéticos. Diário Oficial da União, Poder Executivo, Seção 1, p. 76. Brasília- DF.

Mindelo, et al. (2019) Determinação por titulação do teor de ácido acético em vinagres comerciais coletados em Castanhal-PA. IV Congresso Internacional das Ciências Agrárias. 6(3-5).

Cordeiro, K. L. G. \& Fregonad, D. O., \& Leão V. G. (2019). Teor de ácido acético em vinagres comercializados em ouro preto do Oeste-RO: detecção potenciométrica, colorimétrica direta e de retorno, retrotitulação e condutométrica. Monografia (Graduação). Inter- American Journal of Developmente and Research, 2(1), 11-23.

Costa B. J. P. (2017). Controle de qualidade de produção de vinagre da empresa produtos cheiro verde. 35 p. Monografia. Universidade Federal Rural do semiárido (UFERSA), Centro de Engenharia, Bacharel em engenharia Química. Mossoró-RN.

Dutra J. M. (2021) Produção e caracterização físico-química e microbiológica do vinagre de jambolão (Syzygium cumini). 55. Dissertação (Mestrado).Programa de Pós-Graduação em Tecnologia de Alimentos do Instituto Federal de Educação, Ciência e Tecnologia; Campus Rio Verde Instituto Federal Goiano. Goiás.

Fiorio, J..L., \& Dalposso, P. V (2011). Caracterização e fermentação alcoólica de uva-do japão (hovenia dulcis t.) visando produção de vinagre. 54 p. Monografia (Pós-Graduação). Universidade Tecnológica Federal do Paraná (UTFPR); Campus Pato Branco-Paraná.

Gourmet C. ANAV- Associação Nacional das Indústrias de Vinagre. http://anav.com.br/

Lima K. P (2014). Produção de vinagre como estratégia de aproveitamento tecnológico da amora-preta: avaliação do processo submerso e do processo lento. p.93-113. (22a ed.), 540. Monografia (Mestrado), Processos Bioquímicos da Universidade Tecnológica Federal do Paraná, Pato Branco.

Gusmão, et al. (2020). Análise comparativa do teor de diferentes marcas de vinagres comercializadas no Brasil frente ao especificado no rótulo. Revista Brasileira de Ciências Biomédicas, 1(3). 
Machado, A. et al. (2021). Produção de fermentado acético pelo método submerso. Global Science And Technology, 12(1), 01-13. Universidade Federal de Goiás.

Maestre K. L. (2017) Estudo de condições de fermentação alcoólica e acética utilizando subproduto lácteo e diferentes leveduras. Dissertação (Mestrado) Programa de Pós-Graduação. Universidade Estadual do Oeste do Paraná. Engenharia Química, área de concentração em Processos Químicos e Bioquímicos, Toledo- Paraná.

Marques, F. P. P, et al. (2010) Padrões de identidade e qualidade de fermentados acéticos comerciais de frutas e vegetais. In Food Science and Tecnology, 30(Supl.1): 119-126 Campinas.

Mota, A. C. L. G. (2011) Atividade Antifúngica dos vinagres de álcool e maçã sobre espécie de Candida envolvidas com infecções na cavidade bucal. Dissertação (Mestrado em Estomatologia) Programa de Pós-Graduação em Odontologia do Centro de Ciências da saúde da Universidade. Universidade Federal de Paraíba

Oliveira, N. S. (2014). Desenvolvimento de método não titulométrico para determinação de acidez em amostras de vinagre. p.68-71. Dissertação (Mestrado) Programa de Pós-Graduação em Agroquímica. Universidade Federal de Viçosa - Minas Gerais. CDD (22a ed.), 664-55.

Schmoeller, R. K. \& Balbi, M. E. (2010). Caracterização e controle de qualidade de vinagres comercializados na região metropolitana de Curitiba/PR. Monografia (Graduação) Universidade Federal do Paraná, Visão Acadêmica, 11(2).

Vanin, A. M. et al. (2012). Atividade antioxidante e perfil fenólico de diferentes tipos de vinagres comercializados na região sul do brasil. Monografia (Graduação em Nutrição) Universidade de Caxias do Sul, 23(2), 251-257.

Venquiaruto, L. D. et al. (2016). Teor de ácido acético em vinagres artesanais n região do alto Uruguai Gaúcho. Revista Eletrônica de Extensão da URI. 11(23), 241-246.

Viroli, S. L. M., et al. (2021). Caracterização do vinagre artesanal produzido com casca de maçã. Revista Research, Society and Development, 10(9), e1110917865 